\title{
Les crocs du vampire : mythes et réalités
}

\section{RÉSUMÉ}

Julie NOBELEN

Docteur en chirurgie dentaire,

Lauréat de l'Académie Nationale

de Chirurgie Dentaire.

C.H.U. de Bordeaux,

Pôle d'Odontologie et de Santé buccale,

Hôpital Pellegrin,

Place Amélie-Raba-Léon,

33076 Bordeaux,

France.

\section{Fabienne JORDANA}

\section{Ex Assistant hospitalo-universitaire,}

HIA Robert Picqué,

351 , route de Toulouse,

CS 80002 ,

33882 Villenave d'Ornon,

France.

\section{Jacques COLAT-PARROS}

Maître de Conférences

Université Bordeaux 2,

Praticien hospitalier,

C.H.U. de Bordeaux,

Pôle d'Odontologie et de Santé buccale,

Hôpital Pellegrin,

Place Amélie-Raba-Léon,

33076 Bordeaux

France.

Le vampire appartient à la mythologie universelle des revenants. Sa description a évolué au cours des siècles notamment avec l'apparition de crocs. Récemment, il y a eu résurgence de ce mythe dans le cinéma.

Du mythe à la réalité, il n'y a qu'un pas, franchi par une subculture émergente, les vampyres qui portent des crocs amovibles comme signe de reconnaissance. Les crocs servent également comme déguisement ou lors de jeux de rôles.

Aux États-Unis, certaines officines se sont développées sur un modèle de réalisation directe. En France, la diffusion est restée très confidentielle.

Nous illustrons les étapes de réalisation de crocs dans le cadre d'une demande affirmée et d'une coordination entre cabinet dentaire et laboratoire de prothèse. Le confort de l'utilisateur est lié aux paramètres classiques de réalisation prothétique, en particulier de stabilité statique et dynamique.

canine

prothèse dentaire

modification corporelle

- symbolique

vampirisme
AOS 2012;257:29-39

DOI: $10.1051 /$ aos/2012104

(C) EDP Sciences 
$>$

Nombreuses sont les définitions du vampire. Pour J. Goens, le vampire est " un homme mort dont le corps ne se décompose pas et qui sort la nuit pour sucer le sang des vivants " [1]. Le vampire est attaché aux croyances des pays slaves [2]. II absorbe le sang pour perpétuer sa longévité et chaque victime du vampire devient à son tour vampire $[3,4]$. C'est à partir du XVIIII siècle que les trois caractéristiques qui donnent au vampire sa spécificité sont enfin réunies : le vampire est un « revenant en corps " et non un fantôme éthéré ou un démon, il sort la nuit de sa tombe pour sucer le sang des mortels afin de prolonger son existence posthume, enfin ses victimes deviennent à leur tour des vampires après leur mort [5].

Il appartient à une mythologie universelle, celle des revenants. Ce mort-vivant apparaît comme une tentative de matérialisation d'une préoccupation menaçante fondamentale de I'homme : la mort.

À la frontière de la vie et de la mort, de l'humain et de l'inhumain, le vampire engendre une fascination incomparable et s'avère être immortel, du moins dans l'imaginaire collectif. La première image qui vient à l'esprit à l'évocation du mot vampire est un personnage dont les canines acérées sont prêtes à mordre.
II est intéressant de noter que, jusqu'au XIXe siècle, le vampire n'a pas de crocs. Il suce le sang à travers la peau. C'est avec la littérature fantastique du XIXe siècle qu'il acquiert des dents pointues.

Ce sont les dents qui ont bâti l'image du vampire moderne. II semble peu probable que cette représentation eut été aussi forte si les romanciers n'avaient eu l'idée de le doter d'une longue paire de canines [6].

Du mythe à la réalité, il n'y a qu'un pas. Ce pas a été franchi par une subculture souterraine encore émergente : les vampyres. Apparues dans les années 1990, les communautés vampyriques se développent aux États-Unis et en Amérique du Sud, avant de rejoindre l'Europe (Allemagne, Angleterre, Pays-Bas, France essentiellement). Repérables à leurs crocs, leur signe extérieur d'appartenance le plus marquant, les vampyres constituent une espèce à part, sombre et mystérieuse [7].

La porphyrie $[1,8]$, certaines anomalies morphologiques (dents conoïdes) [9] pouvant être liées à des maladies orphelines [10-17] et même certaines mutilations dentaires [18] peuvent faire apparaître des canines en forme de pointe. Nous ne les présenterons pas, n'étant pas le propos de cet article.

\section{Symbolique de la canine}

Les dents sont indissociables du cycle de la vie : l'apparition des dents de lait correspond au sevrage ou séparation symbolique de sa mère, les dents de sagesse annoncent une nouvelle étape, celle où l'enfant est censé devenir responsable; la perte des dents caractérise la vieillesse et l'approche de la mort [2].

La canine est la seule dent à présenter un nom d'animal, le chien. Elles évoquent les crocs des carnivores et des carnassiers. Le terme argotique de " croc » est d'ailleurs parfois utilisé pour se référer aux canines [19]. Ce sont les dents de la bestialité, de la cruauté. Elles sont le symbole de la haine, de la violence, de l'agressivité. Les canines ont un aspect plus menaçant, plus viril.

À cela s'ajoute une impression de ténacité, d'acharnement. « Canin » signifie en effet insa- 
tiable, inextinguible. On peut dire " un appétit canin », " une faim canine ».

Elles se découvrent surtout lors du rictus et de l'effort et peuvent tout aussi bien montrer la colère ou la menace, la haine ou l'agressivité et ainsi découvrir les instincts destructeurs [20]. "Montrer ses dents " est une expression qui y puise son sens. Cet aspect a été longtemps très sérieusement considéré puisque les criminologistes du XIX siècle affirmaient que des canines hypertrophiées prédestinaient leur détenteur à des actes criminels [21].

Dent la plus ressemblante à la dent haplodonte primitive des vertébrés inférieurs, témoin de notre héritage animal, le croc attrape, possède et garde sa proie [18]. Pointue, la canine est une arme redoutable, elle représente la force brutale, la possession, la domination [3,21]. La symbolique de la canine fait de cette dent un emblème d'énergie vitale instinctive. Elle représente une réserve apparente de désirs matériels, de soif de possession, d'ambition, d'acharnement pouvant aller jusqu'à l'agressivité. L'image des dents longues constitue l'expression transmise oralement d'une connaissance oubliée [22]. La signification sexuelle de la canine n'est plus à démontrer [23]. C'est aussi la dent la plus sujette au dimorphisme [24].

\section{Du mythe à la réalité}

\section{Déguisement et jeux de rôle}

Outre les acteurs et comédiens portant des crocs de vampire pour les besoins de leurs rôles, d'autres personnes portent volontiers des dents de vampire.

Tout d'abord, les personnes qui portent des crocs peuvent être des utilisateurs occasionnels qui se déguisent comme à l'occasion d'Halloween : que ce soit en vampire ou toute autre créature, animal ou monstre ayant des crocs.

Il y a ensuite les joueurs de jeux de rôles grandeur nature et en particulier les joueurs de Vampire: The Masquerade (créé en 1990 par l'Irlandais Mark Rein-Hagen pour la compagnie californienne White Wolf, Inc.), mettant en scène des vampires, membres de sectes secrètes aux objectifs variés.

Dans un jeu de rôle grandeur nature, l'objectif est de recréer I'ambiance de I'univers dans lequel est censé se dérouler l'action. Pour cela, les joueurs et les organisateurs peuvent recou- rir à des costumes ou déguisements, du maquillage, des décors ou des accessoires...

Pour mieux entrer dans la peau de leurs personnages, les joueurs se déguisent en vampires et les crocs constituent l'accessoire qui parfait leurs costumes.

\section{Adeptes du «dental tuning»}

Il s'agit de toutes les transformations temporaires (amovibles) ou définitives concernant les dents à des fins esthétiques.

Cela va du strass discret collé sur une dent aux dents de vampires en passant par les grills qui sont des façades métalliques avec ou non incrustations, strass, gravures, sigles... Ces grills étaient autrefois cantonnés au milieu du hiphop et du rap mais deviennent maintenant plus courants.

Ces transformations s'inscrivent souvent dans une logique de modification corporelle ou body art: tatouages, piercings, scarifications, etc. 


\section{Les vampyres}

L'appellation « vampyre » a été popularisée par Father Sebastiaan (New York), fondateur du clan Sabretooth et de I'Ordo Strigoi Vii (ou ordre des vampyres vivants), pour distinguer les membres de sa communauté des créatures surnaturelles de la littérature et du cinéma fantastique. D'autres vampires ont néanmoins choisi de conserver l'orthographe en usage aujourd'hui [7].

Les vampyres pensent que leur condition résulte d'une déficience au niveau des chakras, les points de passage de l'énergie vitale dans le corps humain, qui les oblige à consommer de grandes quantités d'énergie pranique, un terme sanscrit pour l'énergie vitale. Ils ne sucent donc pas le sang des humains mais leur pompent leur énergie vitale [25].

Les crocs amovibles qu'ils portent en guise de signe de reconnaissance symbolisent la bête qui sommeille en chaque être humain. Selon Father Sebastiaan, il y a deux raisons pour porter des crocs : I'aspect esthétique et l'aspect érotique [7].

\section{Des crocs en résine}

Ces accessoires sont fabriqués à partir de résine dentaire dont l'usage normal est destiné aux couronnes et ponts provisoires.

Pour le clinicien, le matériau idéal doit satisfaire aux exigences suivantes [26-31] :

- esthétique satisfaisante (teinte comparable à celle des dents voisines) ;

- sans goût, ni odeur ;

- biocompatibilité avec absence d'irritation pour la pulpe et le parodonte ;

- stabilité dimensionnelle et absence de porosité ;
- stabilité des teintes ;

- résistance aux contraintes mécaniques et à I'usure, vieillissement ;

- compatibilité avec les autres matériaux ;

- faible conductibilité thermique ;

- facilité de mise en œuvre (élaboration, dégrossissage, meulage, polissage, pose, dépose, rebasage) ;

- température de ramollissement très supérieure à la température de tout aliment ou liquide chaud introduit dans la bouche ;

- on peut également ajouter le critère du coût. Toutes ces propriétés ne sont pas aujourd'hui rassemblées en un même matériau et son choix réside dans le meilleur compromis [31].

La raison pour laquelle les résines dentaires actuelles sont plus ou moins limitées au polyméthacrylate de méthyle est que c'est la seule résine mise au point jusqu'à présent qui présente habituellement, avec des techniques relativement simples, un ensemble de propriétés importantes pour une utilisation en bouche sans détérioration [26].

Les résines pour la réalisation de ces crocs sont les mêmes que celles utilisées pour les couronnes provisoires. Meyer les classe en deux types: les résines autopolymérisables pour le travail direct au fauteuil et les résines polymérisant à chaud ou par activation lumineuse [28].

> Type 1 : résines chémopolymérisables (ou autopolymérisables) (préparation extemporanée)

- Résines acryliques et dérives.

- Résines du type diacrylate, analogues à celles des composites.

- Résines épimines (abandonnées à cause des réactions allergiques). 
> Type 2 :

résines thermopolymérisables

et/ou radiopolymérisables

(polymérisation activée

par des radiations - lumière ou UV)

(préparation au laboratoire)

- Résines acryliques et dérives.

- Résines polyuréthanes.

- Résines polycarbonates [29].

\section{Les personnes qui réalisent ces crocs et leurs techniques}

\section{> Les prothésistes dentaires [32]}

Très souvent, les studios de maquillage et d'effets spéciaux font appel à des prothésistes dentaires qui ont l'expérience de la résine et de la fabrication des prothèses. Leur dextérité manuelle, associée aux concepts prothétiques, leur permet de réaliser de meilleurs produits que les artistes spécialisés dans les effets cosmétiques.

Le laboratoire, pour suppléer aux problèmes d'ajustage, crée une voûte universelle. Le receveur de la prothèse pourra l'adapter en fonction de sa propre bouche par la technique suivante :

- du silicone est placé dans la concavité ;

- cette " over-denture » est placée sur les dents pour obtenir une empreinte. Le silicone est assez stable, c'est une des méthodes de rétention.

Généralement, aucune méthode de rétention n'est nécessaire, étant donné que l'ajustement est très précis. Les alternatives sont également de mettre en place un fermoir, ou d'utiliser l'effet de succion d'un palais acrylique.

$S^{\prime}$ il s'agit de dents unitaires, des capuchons seront réalisés avec un ajustement très précis : ils ressemblent à des couronnes provisoires. La dent n'est pas taillée comme pour une couronne. Nul système de rétention n'est utilisé car, avec l'ajustement précis et les frottements, le capuchon tient tout seul sur la dent.

Une autre technique consiste en la réalisation $d$ 'une gouttière thermoformée avec canines rallongées qui viennent par-dessus les canines naturelles. La gouttière étant en antérieur uniquement du côté palatin, la prothèse ne se voit pas. Un laboratoire français a diffusé une publicité proposant aux particuliers la réalisation de ce type d'accessoires sans intervention d'un chirurgien-dentiste. Un kit de prise d'empreinte était envoyé chez le client avec toutes les instructions nécessaires. Ce laboratoire n'ayant jamais répondu à notre demande de renseignements, il nous a été impossible de vérifier la fiabilité de cette méthode : auto-prise d'empreinte par des personnes n'ayant jamais fait cela auparavant, envoi à leur laboratoire donc temps écoulé long entre l'empreinte et la coulée des modèles, teinte que nous présumons choisie à partir de photos.

\section{$>$ Les chirurgiens-dentistes}

Un chirurgien-dentiste peut réaliser des crocs de vampire par la méthode du bloc technique : exactement comme pour une couronne provisoire mais sans préparer la dent. Ou il peut réaliser des crocs de vampire en association avec un technicien de prothèse dentaire.

\section{$>$ Les fangsmiths [25]}

C'est dans les cercles de jeux de rôle et notamment dans les parties de jeu grandeur nature de Vampire: The Masquerade qu'apparaissent les premiers fangsmiths, selon les cas des autodidactes ou d'anciens prothésistes dentaires reconvertis dans la fabrication des crocs. Ils utilisent des dents en forme de crocs achetées à des fournisseurs de dents caricaturées conçues spécialement pour les prothèses d'effets spéciaux. 
A l'aide d'essayages successifs, le fangsmith en modifie la forme à la demande du client si besoin (longueur, largeur, épaisseur).

En rebasant ce croc avec de la résine acrylique chémopolymérisable, il l'adapte à la dent du client qui obtient donc des crocs réalisés "sur mesure $"$.

La finition se fait par l'ébarbage des excédents de résine et par le polissage des crocs.

\section{Cas clinique}

Nous nous sommes penchés sur une technique indirecte (empreinte et traitement au laboratoire). Nous avons réalisé des crocs simples (canines supérieures) pour illustrer l'aspect classique du vampire et des crocs doubles (incisives latérales et canines supérieures) en raison d'un meilleur résultat esthétique puisque notre sujet a de petites incisives latérales en comparaison de ses incisives centrales, caractéristiques de la denture féminine.

\section{$>$ Protocole}

\section{- Première étape clinique}

- Empreintes précises des deux arcades dentaires à l'alginate : I'enregistrement des faces occlusales et de la ligne du feston gingival sont primordiaux).

- Choix de la teinte (teintier Vita ${ }^{\circledR}$ Lumin Vacuum).

Le choix se porte sur une teinte plus saturée que pour une canine « humaine ».

\section{- Étape laboratoire}

- Coulée des modèles en plâtre pierre extra-dur, type IV (Suprastone de Kerr).

- Montage des modèles obtenus sur articulateur programmable type Fag 2000 et d'un arc facial.

- Montage en cire (wax-up) pour les deux types d'accessoires retenus (cire à modeler de précision $\mathrm{K} 2$ exact de Bredent) :

- deux canines supérieures en 13, 23 ;

- deux canines associées à deux incisives latérales supérieures en 13-12, 22-23 (fig. 1 et 2).

La difficulté pour le technicien de laboratoire a été dans un premier temps de faire abstraction de la morphologie humaine et de tendre vers la forme animale de crocs, en s'inspirant notamment des félins (lion, chat, tigre à dents de sabre...). Puis, dans un deuxième temps, il a fallu resituer cette morphologie dans le contexte humain (occlusion, rapports avec les dents adja-

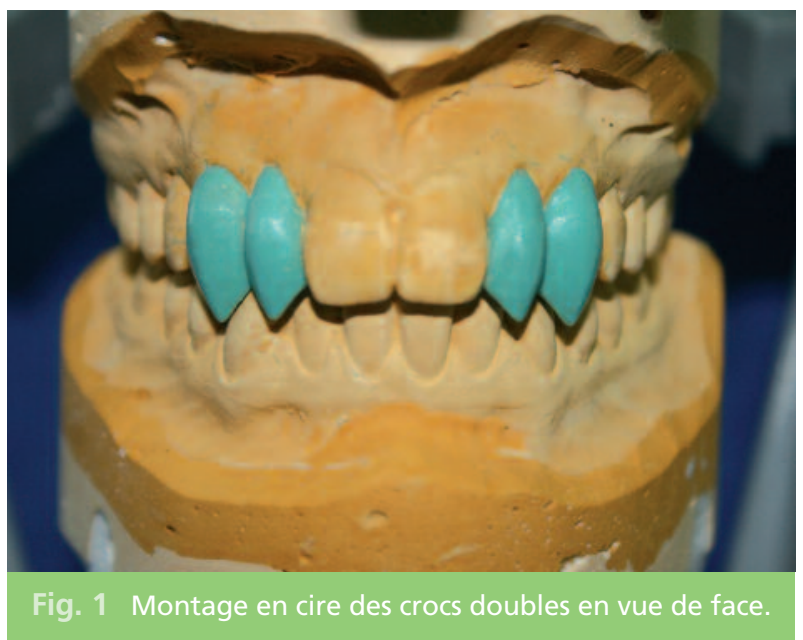



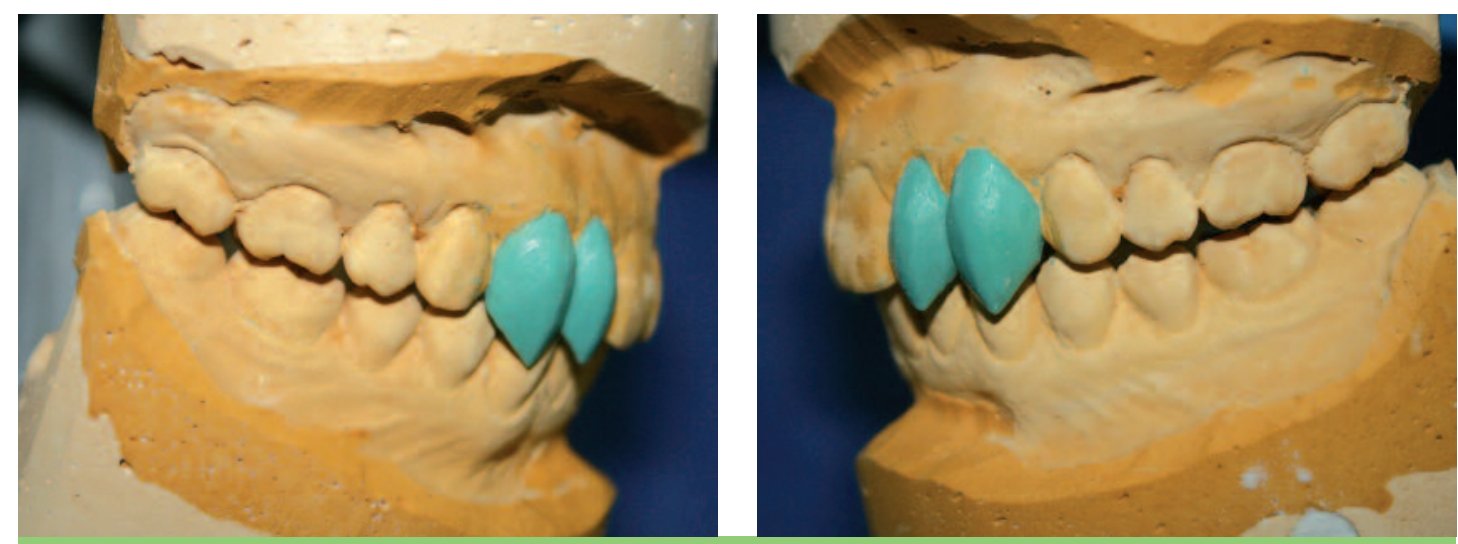

Fig. 2 a et b Montage en cire des crocs doubles en latéralité droite (a) et en latéralité gauche (b).

centes). Le porteur ne doit pas, en effet, être gêné par le port prolongé de cette " prothèse ». La canine humaine présente une pointe cuspidienne, légèrement rentrée et mésialée. La pointe de la cuspide est arrondie sur les dents jeunes, sur les dents plus âgées, elle est remplacée par une petite surface plate, plus ou moins large, provoquée par l'usure [33]. La convexité maximale est située au tiers cervical.

Le croc animal possède une cuspide centrale, très apparente. L'arête vestibulaire, très proéminente, sépare distinctement deux versants du collet à la pointe cuspidienne. La convexité maximale est plus haute que pour la canine humaine.

L'adaptation est plus complexe que pour la réalisation d'une prothèse transitoire classique :

- la prothèse ne doit pas blesser ;

- le croc ne doit pas être visible quand les lèvres sont fermées, mais être apparent dès le début de l'ouverture buccale ;

- la dent n'étant pas préparée, la rétention du croc est réalisée par le bombé du collet vestibulaire et par la face palatine. Les faces proximales sont très fines. Le croc est donc constitué par une fine pellicule de résine. L'ajustage de l'occlusion, plus difficile, doit donc être parfait. $\square$ Réalisation d'une clé en silicone sur le modèle (Zetalabor et Indurent gel C-silicone de Zhermack $\left.{ }^{\circledR}\right)$.

- La clé est désolidarisée du moulage puis des aménagements sont réalisés : les pertuis d'évacuation (fig. 3).

- La clé en silicone est réessayée afin de s'assurer de son positionnement aisé (penser à enlever les languettes interdentaires au niveau

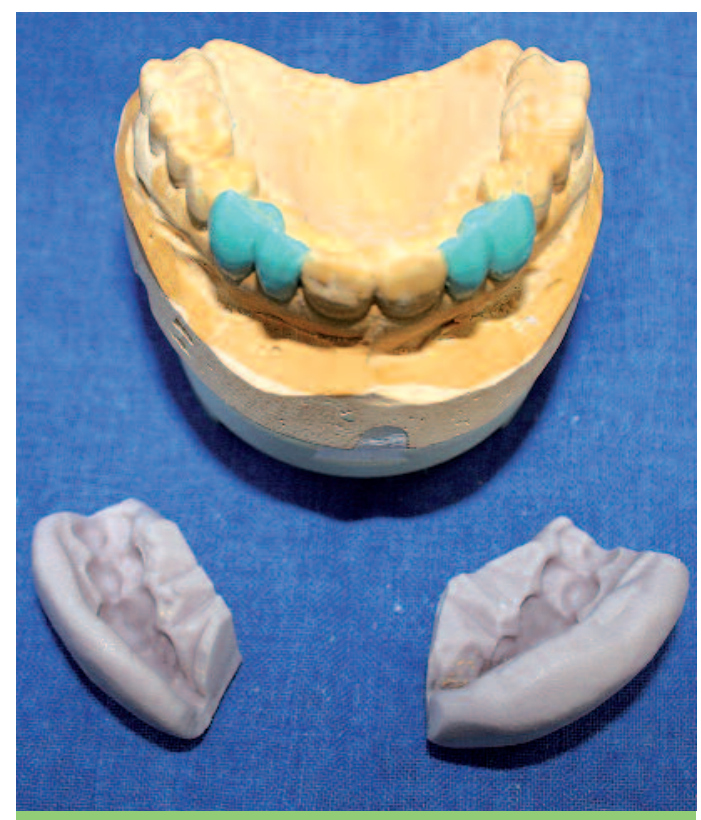

Fig. 3 Clés en silicone des crocs doubles. 
des dents non concernées) et de sa mise en place correcte (pas de bascule, appui d'une à deux dents au moins de chaque côté de la ou des dents concernées).

- La cire du moulage est éliminée par ébouillantage, puis un liquide séparateur est déposé sur les préparations, les dents adjacentes et les surfaces en plâtre.

- La résine acrylique (SR Ivocron de Ivoclar Vivadent ${ }^{\circledR}$ ) est préparée dans un godet et disposée à l'état semi-liquide à l'intérieur des clés en silicones, dans le logement de la (ou des) dents concernée(s). Les clés sont alors installées bien en place, éventuellement maintenues par des élastiques.
- L'ensemble est plongé dans un thermopolymérisateur sous pression pendant 20 minutes dans une eau à $50^{\circ} \mathrm{C}$ (processus contrôlé automatiquement).

- Les clés sont ensuite retirées du moulage.

- Ébarbage.

- Polissage.

- Équilibration sur articulateur.

- Seconde étape clinique (fig. 4 à 6)

- Essayage des crocs simples réalisés.

- Essayage des crocs doubles réalisés.

- Les prothèses tiennent uniquement par rétention. Un rebasage peut être réalisé en bouche si besoin.
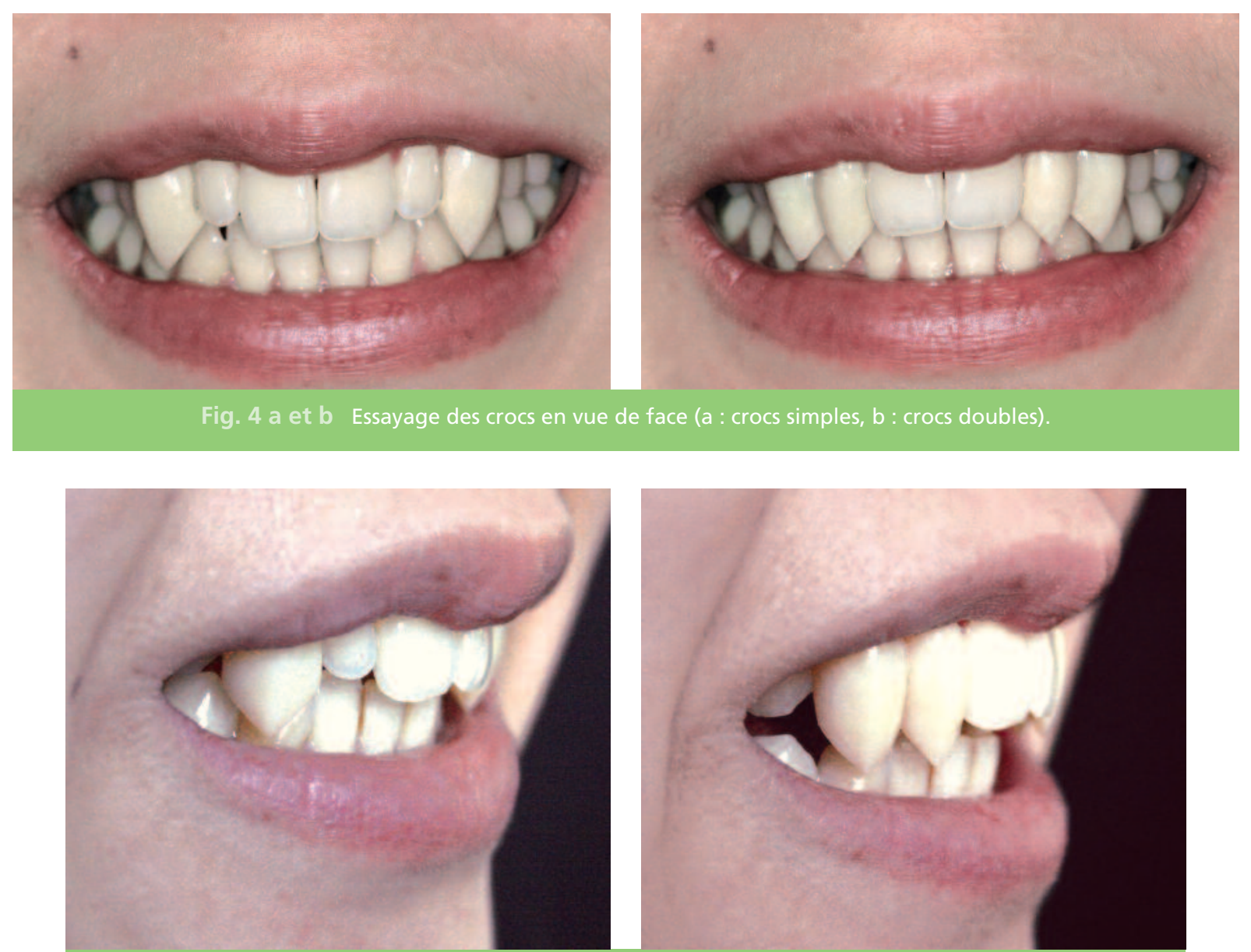

Fig. 5 a et b Essayage des crocs en vue latérale droite (a : crocs simples, b: crocs doubles). 

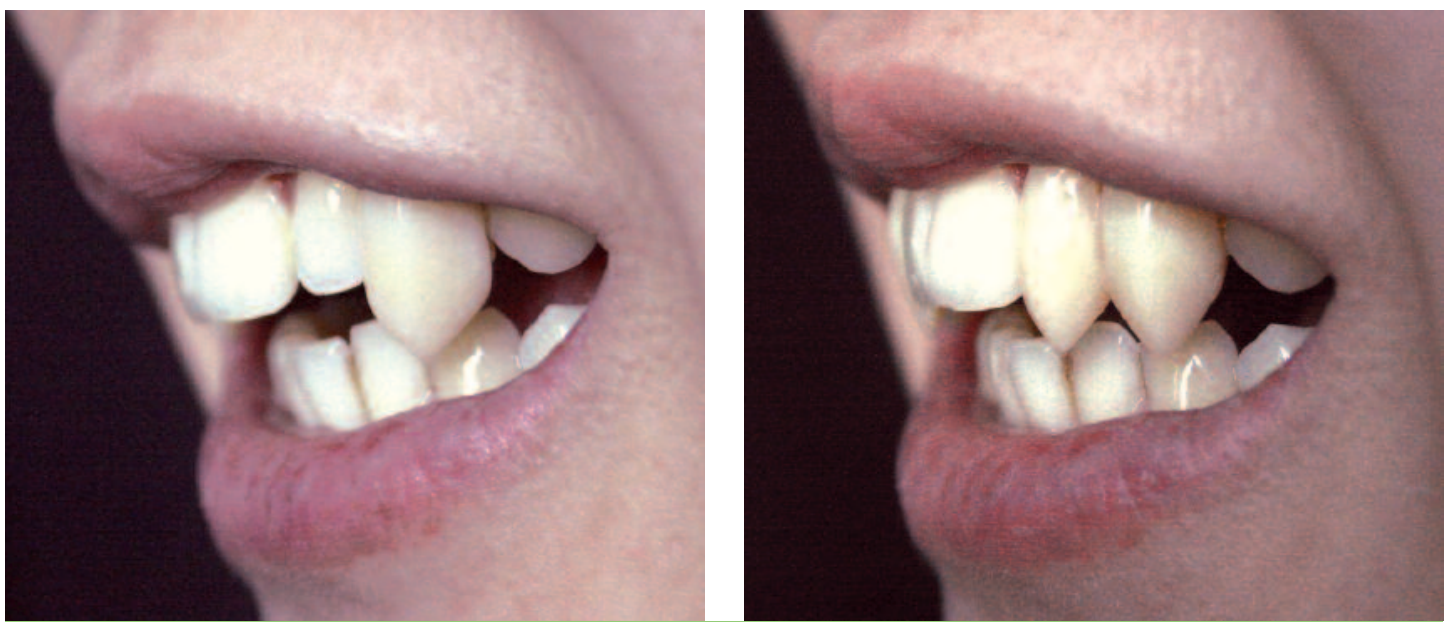

Fig. 6 a et b Essayage des crocs en vue latérale gauche (a : crocs simples, b : crocs doubles).

\section{Conclusion}

Certains patients sont demandeurs de ces accessoires type crocs réalisés dans un seul but esthétique, comme les bijoux dentaires.

Cet article illustre les étapes cliniques et les étapes de laboratoire nécessaires à la réalisation des crocs. Leur réalisation devrait préférentiellement être effectuée par un chirurgien-dentiste pour éviter tout traumatisme sur les dents supports, au niveau des tissus mous et des articulations temporo-mandibulaires. Même si le chirurgien-dentiste est celui qui agira dans les meilleures conditions possibles (matériaux, hygiène, prévention, conseils et suivi), la réalisation d'accessoires de type crocs est exclue de sa capacité professionnelle. En effet, en mars 2010,
l'Ordre national des chirurgiens-dentistes précisait pour la pose de bijoux ou strass que les termes de " prévention ", « diagnostic » et « traitement des maladies [...] » de I'article L4141-1 du CSP excluait toute activité esthétique ne présentant aucune finalité thérapeutique [34, 35].

En revanche, nous avons constaté que les personnes désirant porter des crocs ne pensent pas à en faire la demande auprès de leur chirurgien-dentiste, ou n'osent pas le faire par peur d'un jugement négatif et surtout par peur d'être incompris.

Il faut prendre en compte les risques et les complications au niveau des dents, des tissus mous et des articulations temporo-mandibulaires.

\section{Remerciements}

Abdel Sérier, technicien de laboratoire (CHU de Bordeaux, Pôle d'odontologie et de santé buccale, Hôpital Pellegrin, place Amélie-Raba-Léon, 33076 Bordeaux). 


\section{Bibliographie}

1. Goens J.

Loups-garous, vampires

et autres monstres.

Paris : CNRS Éditions, 1993.

2. Mozzani E.

Livre des superstitions :

mythes, croyances

et légendes.

Paris : Robert Laffont, 1998.

3. Pozzuoli A.

Dracula le centenaire.

Paris : Hermé, 1996.

4. Villeneuve R.

Loups-garous et vampires.

Paris : J'ai Lu, 1960.

5. Marigny J.

Sang pour sang :

le réveil des vampires.

Paris : Gallimard, 1993.

6. Bourbonnais $\mathrm{E}$.

Le symbolisme de la dent

au travers

de quelques mythes

(vampire, loup-garou...)

dans leur expression orale,

littéraire et picturale.

Thèse Doct Sci Odontol.

Nancy : Univ H. Poincaré, 2000.

7. Courau L.

Mutations pop

et crash culture :

une anthologie

de la spirale.org.

Rodez : Éditions

du Rouergue, 2004.

8. Marieb EN, Lachaine R.

Biologie humaine :

anatomie et physiologie.

Paris : De Boeck Université, 2000.

9. Lautrou A.

Anatomie dentaire (2e éd.).

Paris : Masson, 1998.

10. Piette E, Goldberg M.

La dent normale

et pathologique.
Paris : De Boeck Université, 2001.

11. lida $A$, Narai $S$, Takagi $R$, Ono K, Ikeda N.

Blepharo-cheilo-dontic (BCD) syndrome: case report. Cleft Palate Craniofac J 2006;43(2):237-43.

12. Gorlin RJ, Zellweger $\mathrm{H}$, Curtis MW, Wiedemann HR, Warburg M, Majewski F, et al.

Blepharo-cheilo-dontic (BCD) syndrome.

Am J Med Genet 1996;65(2):109-12.

13. Arenas-Sordo $M-L$, Vallejo-Vega B,

Hernández-Zamora E,

Gálvez-Rosas A,

Montoya-Pérez LA.

Incontinentia pigmenti (IP2):

familiar case report

with affected men.

Literature review.

Med Oral Patol Oral Cir Bucal 2005;1(10 Suppl 2):e122-9.

14. Bentolila R, Rivera $H$, Sanchez-Quevedo MC. Incontinentia pigmenti:

a case report.

Pediatr Dent

2006;28(1):54-7.

15. Domínguez-Reyes $A$,

Aznar-Martin T,

Cabrera-Suarea E.

Características generales

y estomatológicas

del síndrome

de Bloch-Sulzberger.

Revisión de la literatura

y aportación

de un caso clínico.

Med Oral 2002;7(4):293-7.

16. Hedge SK, Bhat SS,

Soumya S, Pai D.

Incontinentia pigmenti.

$J$ Indian Soc Pedod Prev Dent 2006;24(Suppl 1):S24-6.
17. Van den Steen $E$, Bottenberg P, Bonduelle M. Dental anomalies associated with incontinentia pigmenti or Bloch-Sulzberger syndrome.

Rev Belge Med Dent 2004;59(2):94-9.

18. Fronty $P$, Sapanet $M$, Georget C, Collet G. L'identification estimative (1re partie).

Poitiers : Éditions Atlantique, 2005.

19. Fronty $P$.

Plaidoyer pour la canine.

Chir Dent Fr 1995;751:29-30.

20. Remaud D.

La dent symbole.

Thèse Doct Sci Odontol. Nantes : Univ, 1989.

21. Loux F.

L'ogre et la dent.

Pratiques et savoirs

populaires relatifs aux dents.

Paris : Berger-Levrault, 1981.

22. Lejoyeux J. Les 9 clefs du visage. Paris : Solar, 1991.

23. Caffin M.

Mon dieu, la dent et moi. Paris : Guy Trédaniel, 2003.

24. Fronty $\mathrm{P}$.

Aspects anthropologique et génétique du dimorphisme sexuel dentaire.

Cah Soc Anthropol Génét Dentofac 1979;5:11-24.

25. Courau L.

Vampyres : quand la réalité dépasse la fiction. Paris : Flammarion, 2006.

26. Skinner EW, Philips RW. Sciences des matériaux dentaires ( $6 \mathrm{e}$ éd.).

Paris : Julien Prélat, 1971. 
27. Krug RS.

Temporary resin crowns and bridges.

Dent Clin North Am 1975;19:313-20.

28. Meyer J-M, Belser U. Les matériaux pour couronnes et ponts provisoires. Réal Clin 1994;5(1):15-24.

29. Malquarti G, Comte $B$ Allard Y, Martin JP, Bois D. Prothèse provisoire immédiate. Encycl Méd Chir Odontologie 1998;23-272-B-10.

30. Morenas M, Deschaumes $C$, Compagnon D.
Prothèse fixée transitoire et biomatériaux : état actuel des connaissances.

Cah Prothèse 1998;104:5-14.

31. Viennot $S$, Malquarti G, Guiu C, Pirel C. Prothèse fixée de temporisation.

Encycl Méd Chir

Odontologie 2007;23-272-B-20.

32. Joslin J, Dempster S. Moulages vivants et effets spéciaux prothétiques. Technologie Dentaire 2002;27(185):37-45

33. Marseillier E, Frison L. Les dents humaines : morphologie.

Paris : Gauthier-Villars, 2004.

34. Ordre National des Chirurgiens-Dentistes. Capacité professionnelle. Pas de strass au cabinet dentaire ! La Lettre Ordre Nat Chir Dent 2010; 85:14.

35. Fronty $Y$, Jordana $F$. Capacité et compétences du chirurgien-dentiste : distinction, étendue et limites.

L'exemple de la chirurgie reconstructrice préet péri-implantaire orale. Encycl Méd Chir Medecine buccale 28-265-I-10 (sous presse).

\section{SUMMARY}

\section{The Vampire's Fangs: Myths and Realities}

Julie NOBELEN,

Fabienne JORDANA, Jacques COLAT-PARROS

\section{Keywords \\ - canine tooth \\ - dental prosthesis \\ - body modification \\ - symbolics \\ - vampirism}

The vampire belongs to the universal mythology of ghosts. The description of the vampire has changed over the centuries with the appearance of fangs and there has been a recent re-emergence of this myth in the cinema.

There is only one step from myth to reality, and this step has been taken by an emerging subculture, the vampyres, with removable fangs as their sign of recognition. The fangs are also used as a disguise or for role playing.

In the USA, some companies have developed on the model where the fangs are made directly by the patient. In France, distribution remains for a very limited clientele only.

We illustrate the steps in producing fangs in the context of an asserted demand and of the coordination between the dental surgery and the prosthetic laboratory. User comfort depends on the classic parameters of prosthesis production, particularly those of static and dynamic stability. 